\title{
Blockage of NMDA- and GABA(A) Receptors Improves Working Memory Selectivity of Primate Prefrontal Neurons
}

\author{
Paul Rodermund, Stephanie Westendorff, and ${ }^{\circ}$ Andreas Nieder \\ Animal Physiology, Institute of Neurobiology, University of Tübingen, D-72076 Tübingen, Germany
}

The ongoing activity of prefrontal neurons after a stimulus has disappeared is considered a neuronal correlate of working memory. It depends on the delicate but poorly understood interplay between excitatory glutamatergic and inhibitory GABAergic receptor effects. We administered the NMDA receptor antagonist MK-801 and the GABA(A) receptor antagonist bicuculline methiodide while recording cellular activity in PFC of male rhesus monkeys performing a delayed decision task requiring working memory. The blockade of GABA(A) receptors strongly improved the selectivity of the neurons' delay activity, causing an increase in signal-to-noise ratio during working memory periods as well as an enhancement of the neurons' coding selectivity. The blockade of NMDA receptors resulted in a slight enhancement of selectivity and encoding capacity of the neurons. Our findings emphasize the delicate and more complex than expected interplay of excitatory and inhibitory transmitter systems in modulating working memory coding in prefrontal circuits.

Key words: GABA; glutamate; iontophoresis; pharmacology; PFC; working memory

Significance Statement

Ongoing delay activity of prefrontal neurons constitutes a neuronal correlate of working memory. However, how this delay activity is generated by the delicate interplay of synaptic excitation and inhibition is unknown. We probed the effects of excitatory neurotransmitter glutamate and inhibitory neurotransmitter GABA in regulating delay activity in rhesus monkeys performing a delayed decision task requiring working memory. Surprisingly, the blockade of both glutamatergic NMDA and GABA(A) receptors improved neuronal selectivity of delay activity, causing an increase in neuronal signal-to-noise ratio. Moreover, individual neurons were similarly affected by blockade of both receptors. This emphasizes the delicate and more complex than expected interplay of excitatory and inhibitory transmitter systems in modulating working memory coding in prefrontal circuits.

\section{Introduction}

Working memory, the ability to retain, manipulate, and recall information after ceasing of its sensory trace, is a vital cognitive function for everyday behavior. Lesion studies in nonhuman primates have established that the PFC is obligatory for working memory in delayed response tasks (Blum, 1952; Mishkin, 1957). A physiological correlate of working memory is persistent activity of neurons in the PFC after removal of the sensory stimulus (Goldman-Rakic, 1996; Dash et al., 2007; Fuster, 2015) and maintenance of behavioral principles (Miller et al., 2003; Vallentin et al., 2012; Eiselt and Nieder, 2013). The persistent activity of these neurons is stimulus specific, commonly leading to elevated responses to a preferred memorandum and inhibitory responses

\footnotetext{
Received Aug. 19, 2019; revised Dec. 3, 2019; accepted Dec. 5, 2019.

Author contributions:P.R. and A.N. designed research;P.R. performed research;P.R. and S.W. analyzed data;P.R. wrote the first draft of the paper; P.R. and A.N. wrote the paper; S.W. and A.N. edited the paper.

This work was supported by Deutsche Forschungsgemeinschaft Grant NI 618/5-2 to A.N.

The authors declare no competing financial interests.

Correspondence should be addressed to Andreas Nieder at andreas.nieder@uni-tuebingen.de.

https://doi.org/10.1523/JNEUROSCI.2009-19.2019

Copyright $\odot 2020$ the authors
}

to nonpreferred ones (Goldman-Rakic, 1999). It has been suggested that the persistent activity of excitatory glutamatergic pyramidal cells found in cortical layer III of the lateral PFC of nonhuman primates arises from recurrent excitation of neurons tuned to the same memorandum (Goldman-Rakic, 1995). These pyramidal cells are thought to preserve response specificity by lateral inhibition from interneurons (Goldman-Rakic, 1995; Arnsten et al., 2012).

Glutamate and GABA are the most prominent excitatory and inhibitory neurotransmitters, respectively, in the PFC (Dash et al., 2007). One of the important glutamate receptors is the NMDA receptor. At a behavioral level, systemic and local administration of antagonists of NMDA and GABA receptors disrupted working memory performance in rodents and nonhuman primates (Sawaguchi et al., 1989; Cole et al., 1993; Chrobak et al., 2008; Smith et al., 2011; Wang et al., 2013; Auger and Floresco, 2014).

NMDA receptors play a major role in working memory activity (Wang, 1999; Skoblenick and Everling, 2012; Wang et al., 2013). Such effects of NMDA receptors could be mediated by NMDA receptor subtype $2 \mathrm{~B}$ subunits (NR2B) that show slow gaiting kinetics and saturation properties well suited for causing 
sustained elevated firing rates (Wang, 1999, 2001, 2002; Compte et al., 2000; Wang et al., 2013; Wang and Arnsten, 2015). However, the effects of general NMDA antagonists, such as MK-801 (MK), are contradictory. Iontophoretic administration of MK in the PFC of behaving monkeys reduced task-related firing, but not spontaneous activity per se (Wang et al., 2013). While some studies with rodents found that MK preferentially blocked receptors on interneurons, others found stronger effects on pyramidal cells (Jackson et al., 2004; Homayoun and Moghaddam, 2007; Rotaru et al., 2011). As the excitatory effects of MK were only seen after systemic but not after local injection of NMDA antagonists in the rodent PFC, it has been suggested that they are based on blockage of inhibitory interneurons in other brain areas that project to the PFC (Suzuki et al., 2002; Lorrain et al., 2003; Skoblenick and Everling, 2012).

Inhibitory effects of GABA are also thought to play a role in coding working memory and attention (Funahashi et al., 1989; Compte et al., 2000; Yoon et al., 2016; Auger and Floresco, 2017; Bast et al., 2017). Bicuculline methiodide (Bic) is a common GABA(A) antagonist that reduced the tuning of task related neurons in the PFC by elevating the firing rate to nonpreferred stimuli and decreasing signal-to-noise ratio (Rao et al., 2000). However, the behavioral effects elicited by GABA antagonists are not consistent. For example, studies with rodents have shown that GABA antagonists in some cases did not impair working memory, memory acquisition, or retention learning, and had a supportive effect on memory consolidation (Luft et al., 2004; Kim et al., 2012; Auger and Floresco, 2014; Farahmandfar et al., 2017).

To investigate the effects of glutamate and GABA on neuronal response properties in the PFC, we iontophoretically blocked NMDA and GABA(A) receptors while recording from PFC neurons of macaques performing a perceptual decision task. In this task, the monkeys had to decide and memorize over a delay period whether or not they had seen a stimulus displayed with different near-threshold intensities (Merten and Nieder, 2012). Based on the previous literature, we hypothesized that both MK and Bic would decrease the coding capacity of delay-selective PFC neurons. However, we observed the opposite effect.

\section{Materials and Methods}

Subjects and surgery. We trained 2 male rhesus monkeys (Macaca mulatta) on a delayed perceptual decision task. Monkey Q was 7 years old and weighed $8.5 \mathrm{~kg}$; Monkey $\mathrm{Z}$ was 9 years old and weighed $8.1 \mathrm{~kg}$. Monkeys were implanted with titanium head posts for head fixation and a recording chamber above the right lateral PFC, centered over the principal sulcus (see Fig. 1d). Surgery was conducted using aseptic techniques under general anesthesia. Structural MRI was performed before implantation to locate anatomical landmarks. All procedures were authorized by the relevant authority, the Regierungspräsidium Tübingen, Germany.

Experimental design. Monkeys were trained to report the presence or absence of a visual stimulus by responding to an associated set of response rule-cues varying in color or shape (see Fig. 1a). The sample stimulus consisted of a gray circle $\left(1.5^{\circ}\right.$ of visual angle) presented at six intensity levels. Intensity levels were individually adapted to the monkeys: three sample stimuli were salient and three sample stimuli were around the perceptual threshold of the monkey (see Fig. 1c). Intensity of threshold sample stimuli for Monkey Q were slightly reduced after 14 of 70 recording days to ensure they were still around perceptual threshold. Stimulus intensities are depicted in RGB values, stimulus intensity levels in ordinal numbers, with lower values representing lower stimulus intensities. Given values do not represent actual physical intensity levels of the presented stimuli.

Throughout a trial, the monkeys fixated on a central white fixation $\operatorname{spot}\left(0.1^{\circ}\right.$ of visual angle $)$ and kept their gaze within $3^{\circ}$ of visual angle. Eye movements were monitored with an infrared eye-tracking system (ISCAN). CORTEX program (National Institute of Mental Health) was used for experimental control and behavioral data acquisition.

The animals initiated each experimental trial by grasping a lever and fixating a central fixation target (fixation period). After $500 \mathrm{~ms}$, a visual stimulus (gray circle) was displayed for $500 \mathrm{~ms}$ in half of the trials (stimulus period); in the other half, no stimulus was shown. Both trial types were randomly intermixed. After the delay period (2000 ms), a responserule cue was presented. Two response-rule cues were associated with the presence of a sample stimulus (red square or gray triangle) and two response-rule cues with its absence (blue square or gray cross). If a sample stimulus had been presented, a red square or a gray triangle as response-rule cue required the monkey to release the lever within 1200 ms to receive a fluid reward, whereas a blue square or gray cross as response-rule cue required the monkey to keep fixation and hold on to the lever for another $1200 \mathrm{~ms}$. The associated response rule-cues applied in the inverse way if no sample stimulus had been presented (see Fig. 1b).

Electrophysiology. We performed extracellular single-cell recordings in the right lateral PFC centered on the principal sulcus. In each recording session, up to three custom-made electrode-pipette combinations were inserted transduraly using a modified electrical microdrive (NAN Instruments) (Jacob et al., 2013). Neurons were recorded at random; no attempt was made to preselect neurons according to particular response properties. Signal acquisition, amplification, filtering, and digitalization were accomplished with the MAP system (Plexon). Waveform separation was performed offline (Offline Sorter; Plexon).

Iontophoresis. MK and Bic were applied iontophoretically (MVCS iontophoresis system; npi electronic) using custom-made tungsten-in-glass electrodes flanked by two pipettes each (Thiele et al., 2006). Electrode impedances were usually $\sim 1 \mathrm{M} \Omega$, full range $0.2-6.4 \mathrm{M} \Omega$ (measured at 500 $\mathrm{Hz}$; Omega Tip Z; World Precision Instruments). Pipette resistances depended on the pipette opening diameter, drug, and solvent used. Typical resistances were between 15 and $60 \mathrm{M} \Omega$, full range $10-168 \mathrm{M} \Omega$.

Retention currents were $-7 \mathrm{nA}$ for both drugs. Ejection currents for MK $(0.01 \mathrm{~mol} / \mathrm{L}$ in double-distilled water, $\mathrm{pH} 3.8$ with HCl; Sigma Millipore) were usually at $25 \mathrm{nA}$, full range $15-25 \mathrm{nA}$, which was comparable with Wang et al. (2013). Ejection currents for Bic $(0.002 \mathrm{~mol} / \mathrm{L}$ in doubledistilled water, pH 3.9 with $\mathrm{HCl}$; Sigma Millipore) were usually at $15 \mathrm{nA}$, full range 15-25 nA, which was analogous to Rao et al. (2000). If only one drug was administered per electrode, the other flanking pipette was filled with $0.9 \% \mathrm{NaCl}, \mathrm{pH}$ 7. Electrode impedance and pipette resistance were measured after each recording session.

Trial blocks without drugs alternated with trial blocks during which drugs were continuously applied. Both control and drug blocks lasted between 8 and $26 \mathrm{~min}$, depending on the time the monkeys needed to reach a sufficient number of correct trials. The first block and all oddnumber blocks were control conditions; in the even-number blocks, the drug was administered. In the subset of neurons that were tested with both MK and Bic in sequence, the washout period was the duration of the control blocks (i.e., between 8 and $26 \mathrm{~min}$ ).

In previous iontophoretic experiments using exactly the same apparatus and methods, we have ensured that neuronal effects are not caused by positive ejection currents (Jacob et al., 2013; Ott et al., 2014). In such control experiments with $0.9 \%$ physiological $\mathrm{NaCl}$ and ejection currents of $25 \mathrm{nA}$ (as used here), or even higher values of $50 \mathrm{nA}$, none of the tested neuronal responses (either spontaneous activity or any of the selective responses) was affected by ejection currents alone (Jacob et al., 2013; Ott et al., 2014). In addition, we show here that application of MK and Bic resulted in opposite effects on the neurons' spontaneous firing rates, even though both drugs were ejected by the same amounts of positive currents. Together, this confirms that the effects observed in the present study were caused by pharmacological receptor blockade.

Statistical analysis. Data analysis was performed using MATLAB (The MathWorks). All significance levels were $\alpha=0.05$. Behavioral performance was assessed by signal detection theory, classifying the monkeys' responses as correct (hits and correct rejections) or wrong (misses and false alarms). To obtain psychometric detection curves, we calculated the ratio of correct to wrong responses for each stimulus intensity. We compared psychometric performance using two-way repeated-measures 
a

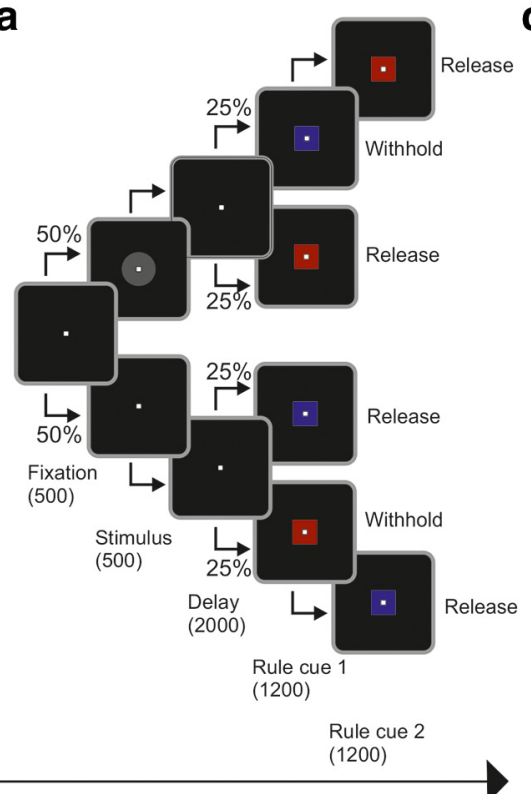

b

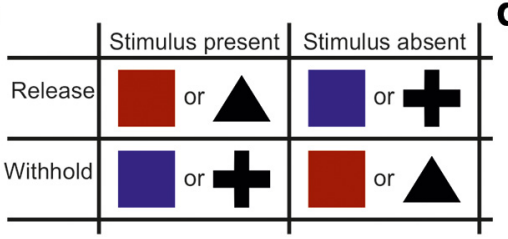

C
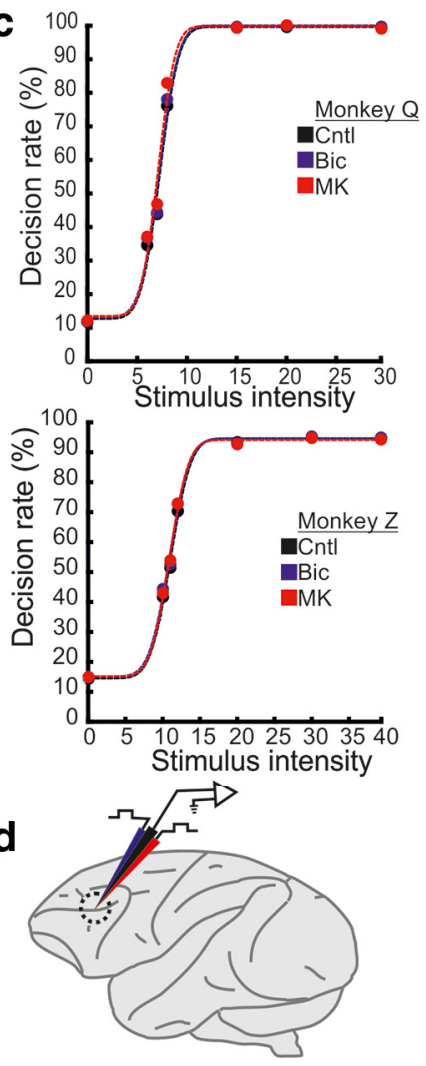

Figure 1. Behavioral protocol, performance, and recording site. $\boldsymbol{a}$, After grasping a lever and holding fixation for $500 \mathrm{~ms}$, a sample stimulus of varying contrast was flashed for $500 \mathrm{~ms}$ in $50 \%$ of the trials (top branch), and no stimulus was shown in the remaining $50 \%$ (bottom branch). After a delay of $2000 \mathrm{~ms}$, one of four target stimuli was shown and, depending on whether or not a sample stimulus was presented, either instructed the monkey to release the bar or to withhold a response. $\boldsymbol{b}$, Instructed response rules (left column) with respect to stimulus condition (middle and right column) by the respective rule cues. $c$, Psychometric detection curves for Monkey Q (top, 70 sessions) and Monkey Z (bottom, 59 sessions) subdivided in control (Cntl), Bic, and MK trials. $\boldsymbol{d}$, Lateral view of a macaque monkey brain. Circled area represents the area of extracellular recording and iontophoresis targeting $G A B A(A)$ and NMDA receptors at the principal sulcus.

ANOVAs with main factors drug condition and stimulus intensity. The amount of aborted trials was compared across drug conditions using Wilcoxon signed-rank tests for paired data.

For neuronal analyses, we sorted spikes offline and studied the responses of all well-isolated neurons. Neurons that had a mean firing rate $>1 \mathrm{~Hz}$ and were recorded for at least 8 trials each when the stimulus was present and absent during control and at least one drug condition were analyzed. For spike density population plots, spike rates were normalized by subtracting the mean baseline firing rate in control trials and dividing by the respective SD.

Neurons were classified as either broad-spiking (BS) or narrowspiking (NS) cells (i.e., putative pyramidal cells or interneurons) with a linear classifier ( $k$-means, $k=2$, squared Euclidean distance) (Diester and Nieder, 2008). Only cells that had a downward deflection in voltage before an upward deflection were classified. More precisely, the minimum of the extracted waveform had to occur between 200 and $400 \mu \mathrm{s}$ and the maximum after $>300 \mu \mathrm{s}$. A total of 49 of 281 units did not fulfill these criteria. Waveforms were normalized by their difference between maximum and minimum voltage deflection and aligned to their minimum.

To examine drug effects on spontaneous firing rates, we compared firing rate of these neurons during the fixation period between control and respective drug condition using a Wilcoxon signed-rank test for paired data. To investigate modulation of spontaneous firing rates at the transition from control to drug phase, we normalized baseline firing rates by dividing with the mean firing rate during control condition. All reported neuronal analyses are based on correct trials only.
As most neurons did not have an elevated firing rate throughout the whole delay period, we used a sliding-window approach. We calculated spike densities by convolving each spike with a Gaussian kernel ( $\sigma=50 \mathrm{~ms}$ ). Thus, $95 \%$ of the area under the Gaussian correspond to a window size of $196 \mathrm{~ms}$. The obtained spike density functions were sampled with 10 ms resolution. Next, we calculated two-way ANOVAs with the main factors stimulus condition (present/absent) and drug condition (control/ drug) for each $10 \mathrm{~ms}$ bin of the spike densities in the delay phase. To ensure that neurons were no longer responsive to presentation of the sample stimulus, we excluded the first $100 \mathrm{~ms}$ of the delay. Cells that selectively responded to the stimulus condition for at least 300 consecutive milliseconds entered further analyses. In other words, the null hypothesis had to be rejected for at least 30 tests in a row. The longest selective time span was used as the analysis window for the respective neuron.

We quantified selectivity of the stimulusselective neurons (defined by the procedure described above) using receiver operating characteristic (ROC) analysis on the firing rates of the neuron for stimulus absent and present trials (Green and Swets, 1966). The area under the ROC curve (AUROC) depicts the discriminability of two distributions, where 1 indicates perfect discriminability and 0.5 signals no separation. Stimulus present and absent conditions were labeled as preferred or nonpreferred based on the respective AUROC values. If a neuron fired more strongly to the stimulus present condition (resulting in an AUROC value $>0.5$ ), the stimulus present condition was the preferred condition. However, if a neuron responded more strongly to the stimulus absent condition, the stimulus absent condition was the preferred condition. For drug trials, we kept the same analysis window and labels regarding stimulus preference as for control trials and calculated AUROC values again. AUROC values in control and drug condition were compared with a paired $t$ test.

We calculated mean firing rates of stimulus-selective neurons during their respective analysis window and compared drug effects on stimulus absence- and presence-preferring neurons with two-way repeatedmeasures ANOVAs with drug condition and stimulus intensity as main factors. Next, we compared mean firing rates and Fano factors for preferred and nonpreferred stimulus conditions across drug conditions with paired $t$ tests (Fano, 1947). Mean firing rate differences between control and drug condition were calculated separately for preferred and nonpreferred stimulus conditions and also compared with a paired $t$ test.

The ROC analysis was repeated for those neurons that were tested in control and both drug (MK and Bic) conditions $(n=53)$. We used a binomial test to examine whether the amount of neurons that increased their AUROC values with administration of either drug was expected by the amount of neurons that increased their AUROC values with one of the drugs.

\section{Results}

We investigated 2 rhesus monkeys trained on a delayed perceptual decision task. At the beginning of a trial, either a sample stimulus of varying intensity was flashed or no sample stimulus was shown (stimulus absent trials) (Fig. 1a). The monkeys had to decide whether or not they had seen a stimulus. Intensity levels of the sample stimuli were individually adapted to the monkeys 

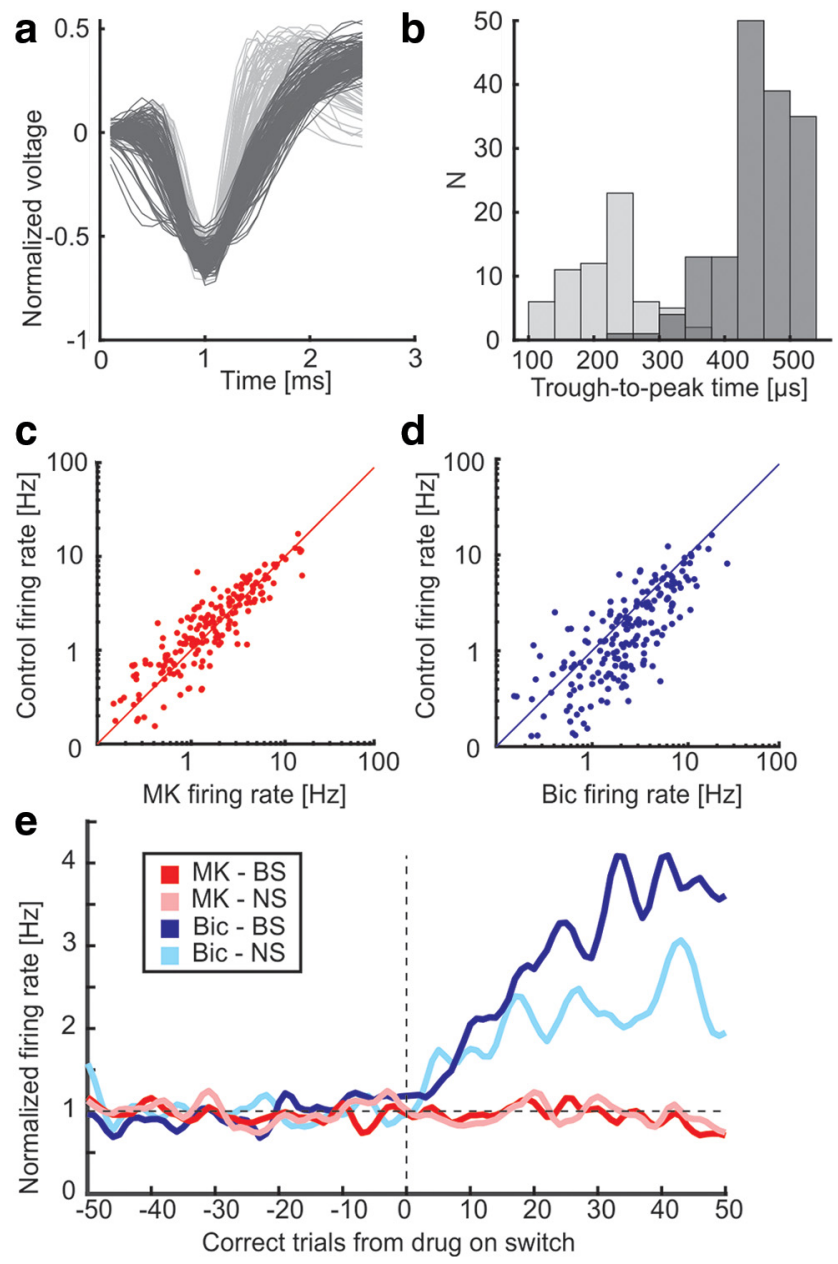

Figure 2. Waveform separation and drug effects on spontaneous firing rate. $\boldsymbol{a}$, Waveforms of recorded neurons. $\boldsymbol{b}$, Distribution of $\mathrm{BS}$ and NS neurons. $\boldsymbol{c}, \boldsymbol{d}$, Spontaneous firing rate during the fixation period in control and drug conditions: $\boldsymbol{c}$, MK, 186 neurons; $\boldsymbol{d}$, Bic, 193 neurons. $\boldsymbol{e}$, Normalized spontaneous firing rate for BS and NS neurons for the last 50 correct trials in control condition before drug on switch and the first 50 correct trials after drug on switch.

such that three sample stimuli were salient (stimulus present trials) and three sample stimuli were around the perceptual threshold (stimulus threshold trials). This challenged the monkeys' stimulus present/stimulus absent judgments and forced subjective decisions. The monkeys maintained their decision in working memory to later report their decisions as a function of subsequent response-rule cues. Importantly, the response-rule cues informed the monkeys whether or not a motor act was required depending on their memorized decisions (Fig. 1b). Neuronal activity in the delay period was thus working memoryrelated and dissociated from potential motor preparation.

The monkeys' behavioral performance was classified according to signal detection theory. Hits and correct rejections were rewarded, whereas misses and false alarms were not reinforced. Not reinforcing misses of stimuli that were presented around perceptual threshold leads to a small bias of the monkeys to erroneously report the presence of a stimulus in some of the stimulus absent trials. Both monkeys were able to detect the salient stimuli in $>90 \%$ of the cases and correctly rejected $>80 \%$ of the trials in which no stimulus was shown. Psychometric detection curves are depicted in Figure $1 c$.

While the monkeys performed the task, we recorded 281 single neurons from the lateral PFC (118 from Monkey Q and 163
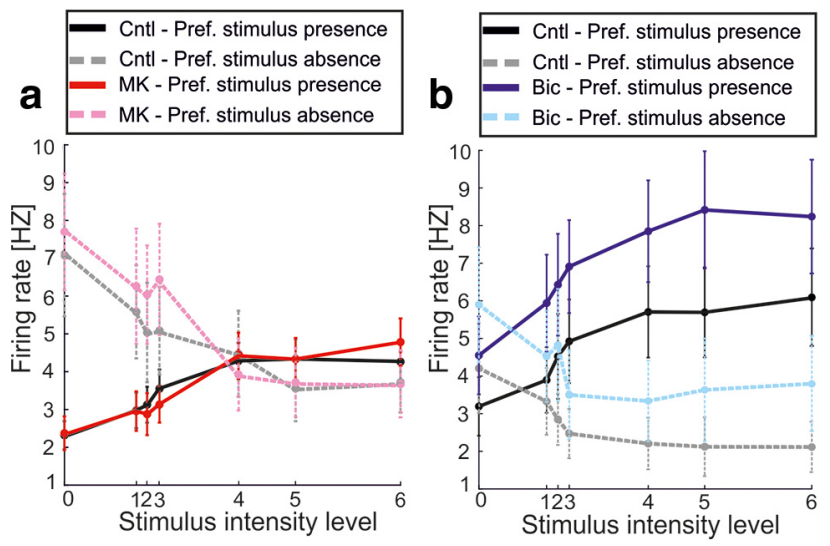

Figure 3. Drug effects on firing rate for neurons preferring stimulus absence or presence. Because the monkeys worked with different absolute intensity values, the data are plotted in relative intensity values according to the monkeys' individual psychometric functions (Fig. 1C). Relative stimulus intensity levels 0 - 6 correspond to absolute stimulus intensities $0,6,7,8,15$, 20, 30 for Monkey $Q$ and $0,10,11,12,20,30,40$ for Monkey $Z$. Firing rate means were derived during each neuron's respective analysis window. $\boldsymbol{a}$, Red represents firing rates with MK relative to control discharges in black and gray. $\boldsymbol{b}$, Blue represents firing rates with Bic relative to control firing rates. Solid lines and darker colors represent neurons preferring stimulus presence. Dashed lines and lighter colors neurons preferring stimulus absence. Error bars indicate SEM.
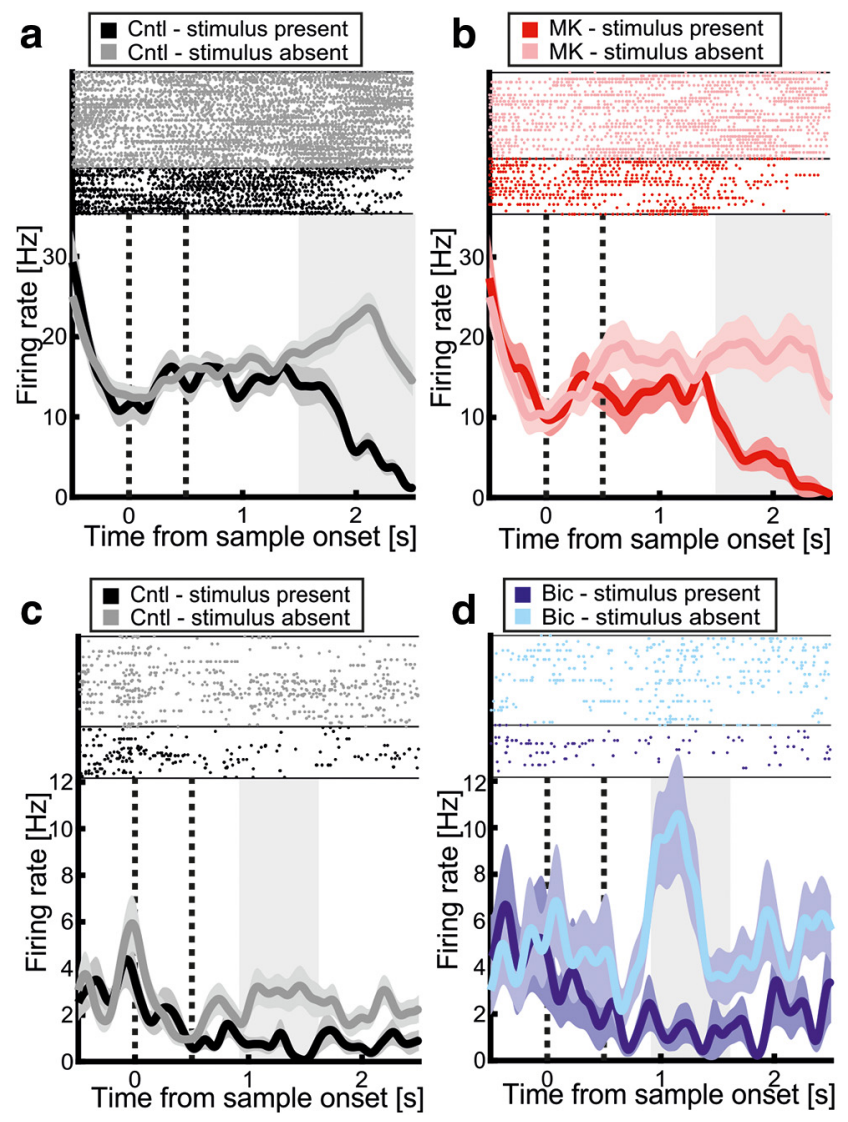

Figure 4. Drug effects on selectivity for example neurons. Raster plots (row represents a trial; dot represents a spike) and spike density histograms (time-resolved average firing rates) representing the activity of an example neuron with increased delay selectivity during MK administration $(\boldsymbol{a}, \boldsymbol{b})$ and an example neuron with increased delay selectivity during Bic administration $(\boldsymbol{c}, \boldsymbol{d})$. Dashed vertical lines indicate beginning of stimulus presentation and delay period. Shaded backgrounds represent the analysis window. Dark colors represent stimuluspresent trials. Light colors represent stimulus-absent trials. Shaded areas around the spike density histograms represent respective SEs. $\boldsymbol{a}, \boldsymbol{b}$, An NS cell preferring stimulus-absent trials from Monkey $Z$ in control $(\boldsymbol{a})$ and MK $(\boldsymbol{b})$ condition. $\boldsymbol{c}, \boldsymbol{d}$, An NS cell preferring stimulus-absent trials from Monkey $Q$ in control $(\boldsymbol{c})$ and Bic $(\boldsymbol{d})$ condition. 

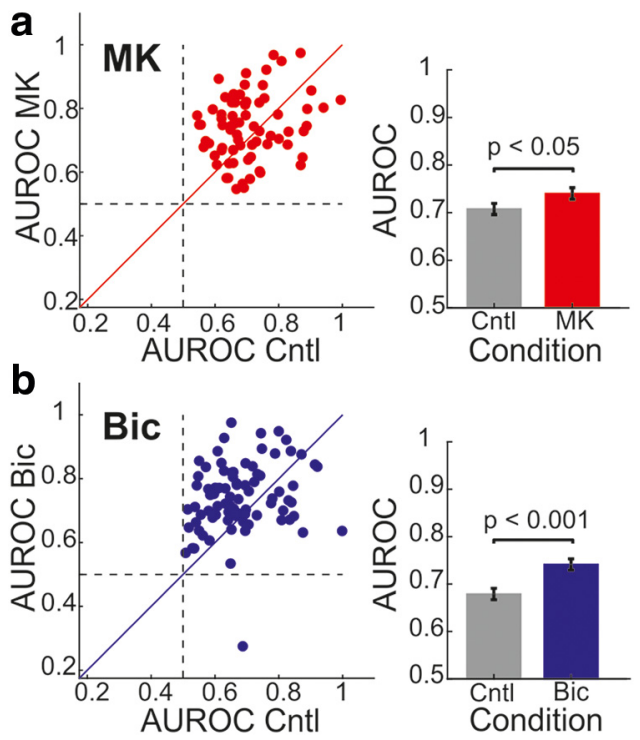

Figure 5. Drug effects on the selectivity for the population of stimulus-selective neurons. AUROC values represent discriminability between preferred and nonpreferred stimulus condition in control and drug condition ( $\boldsymbol{a}$ : MK, 72 neurons; $\boldsymbol{b}$ : Bic, 83 neurons). Left, AUROC values for each neuron. Right, Mean (and SE) for the respective population.

from Monkey Z; Fig. 1d). These neurons were classified into NS and BS neurons based on their waveform characteristics (Diester and Nieder, 2008; Viswanathan and Nieder, 2015). During recordings, trial blocks without pharmacological manipulation (control condition) alternated with blocks in which either MK or Bic was applied to the vicinity of the recorded cells by iontophoresis (drug condition). A total of 186 neurons fulfilled the criteria in control and MK conditions, 193 neurons in control and Bic conditions, and 98 neurons in control and both drug conditions.

We first explored the potential effects of the drugs on the monkeys' behavior. Administration of either drug improved psychometric performance for Monkey Q $\left(F_{(2)}=3.75, p=0.029\right.$, two-way repeated-measures ANOVA), but not for Monkey $\mathrm{Z}$ $\left(F_{(2)}=0.49, p=0.614\right.$, two-way repeated-measures ANOVA) (Fig. 1c). In addition, both monkeys aborted significantly more trials in both drug conditions, independent of the respective stimulus intensity. Specifically, Monkey Q aborted $43.80 \%$ of all trials during MK administration, compared with $20.26 \%$ during respective control trials $(Z=-6.27, p<0.001$, paired Wilcoxon signed-rank test), and $42.31 \%$ during Bic administration, compared with $20.55 \%$ during respective control trials $(Z=-6.27$, $p<0.001$, paired Wilcoxon signed-rank test). Similarly, Monkey $\mathrm{Z}$ aborted $42.96 \%$ of all trials during MK administration, compared with $20.51 \%$ during respective control trials $(Z=-6.15$, $p<0.001$, paired Wilcoxon signed-rank test), and $41.84 \%$ during Bic administration, compared with $20.71 \%$ during respective control trials $(Z=-6.21, p<0.001$, paired Wilcoxon signedrank test).

\section{NMDA receptor blockade reduced spontaneous firing rate, whereas $\mathrm{GABA}(\mathrm{A})$ receptor blockade increased it}

We first evaluated the general effect of MK and Bic on the PFC neurons' spontaneous firing rates. Application of MK slightly decreased spontaneous firing rates $(Z=-2.41, p=0.016$, paired Wilcoxon signed-rank test), whereas Bic significantly increased them $(Z=7.39, p<0.001$, paired Wilcoxon signed-rank test) (Fig. 2c,d). We next separated the neurons into broad-spiking
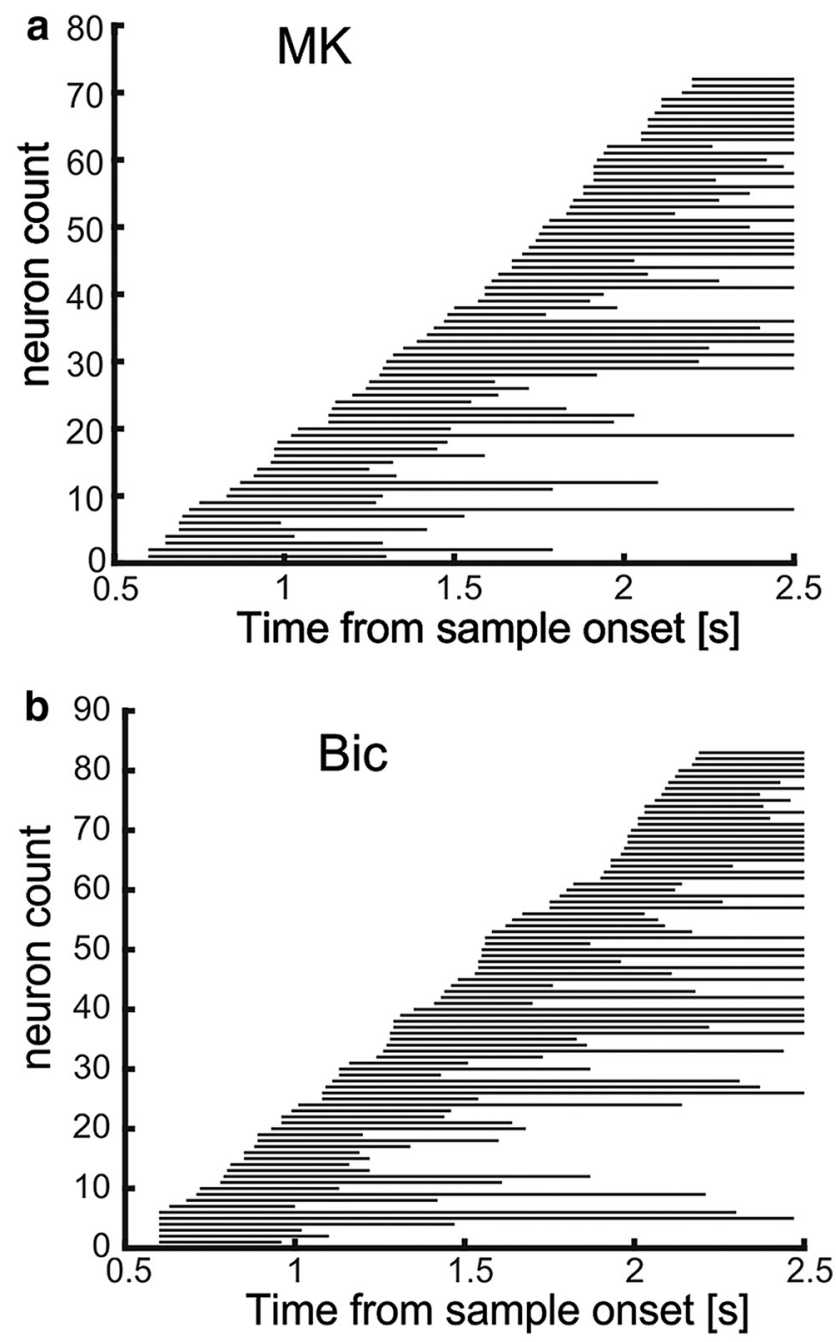

Figure 6. Distribution of analysis windows of stimulus-selective neurons. Stimulus-selective neurons in MK (a) and Bic $(\boldsymbol{b})$ condition are sorted according to the beginning of their analysis window.

(i.e., putative pyramidal cells, BS) and narrow-spiking cells (i.e., putative interneurons, NS) based on the waveform of the extracellularly measured spikes (Fig. $2 a, b$; see Materials and Methods). The effects of MK and Bic were similar for the subpopulation of BS and NS. BS tended to decrease their firing rate with application of MK $(n=106, Z=-1.87, p=0.061$, paired Wilcoxon signed-rank test); NS were not modulated by MK ( $n=46, Z=$ $-1.61, p=0.107$, paired Wilcoxon signed-rank test). Bic increased firing rates for BS $(n=115, Z=6.35, p<0.001$, paired Wilcoxon signed-rank test) as well as for NS ( $n=47, Z=4.42$, $p<0.001$, paired Wilcoxon signed-rank test). The number of BS and NS do not add up to the total of 186 neurons analyzed for MK, and the total of 193 neurons analyzed for Bic, because the waveform of some neurons could not be classified as neither broad nor narrow and were excluded.

Next, we examined the time course of modulation of firing rates at the transition from control to drug phases (Fig. 2e). In the pooled data, drug effects of MK are no longer significant. The continuously increasing firing rate enhancement caused by Bic is clearly visible, and was stronger for BS compared with NS. 
NMDA and GABA(A) receptor

blockade each improved selectivity to preferred stimulus condition

Many of our recorded neurons showed selective activity either for stimulus present trials or stimulus absent trials. We used a sliding window ANOVA to assess selectivity of the neurons to stimulus condition in the delay period (see Materials and Methods) and refer to the stimulus condition that caused a significantly elevated firing rate as the preferred stimulus condition, whereas the other condition is referred to as the nonpreferred stimulus condition. Of 186 neurons recorded in the MK condition, 72 were stimulus-selective; of those, 46 preferred stimulus presence and 26 preferred stimulus absence. Likewise, of the 193 neurons recorded under the Bic conditions, 83 were stimulus-selective; of those, 56 preferred stimulus presence and 27 preferred stimulus absence. Of the 98 neurons recorded in both drug conditions, 53 were stimulus-selective, with 34 preferring stimulus presence and 19 preferring stimulus absence.

Next, we explored the neuromoteric functions with and without drugs for the stimulus-absent and stimulus-present neuron population separately. To that aim, we compared the mean firing rates of neurons preferring stimulus presence or absence in control and drug condition across all stimulus intensities (Fig. 3). Significant firing rate differences after drug applications were observed for both neurons that preferred the stimulus-present condition and neurons that preferred the stimulus-absent condition. Bic, in particular, caused a clear upward parallel shift of the neurometric functions for both cell categories (Fig. 3b).

Specifically, we found no effect of MK on the firing rate of stimulus presence-preferring neurons $\left(F_{(1)}<\right.$ $0.01, p=0.999$, two-way repeated-measures ANOVA), and no interaction of firing rate with intensities $\left(F_{(6)}=1.11, p=0.355\right)$. We also found no effect of MK on the firing rate of stimulus absence-preferring neurons $\left(F_{(1)}=2.30, p=0.142\right.$, two-way repeated-measures ANOVA), but a marginal interaction of firing rate with intensities $\left(F_{(6)}=2.25, p=0.042\right)$. Post hoc testing revealed significant differences at stimulus intensities 3 and 4 . In contrast, we found significant differences in firing rate following Bic administration for stimulus presence-preferring $\left(F_{(1)}=\right.$ $23.51, p<0.001$, two-way repeated-measures ANOVA), without significant interaction $\left(F_{(6)}=1.22, p=0.294\right)$. Similarly, Bic administration significantly changed activity for stimulus absence-preferring neurons $\left(F_{(1)}=5.41, p=0.028\right.$, two-way repeated-measures ANOVA), again without significant interaction $\left(F_{(6)}=0.78, p=0.587\right)$.

We further analyzed the impact of both drugs on the encoding of the stimulus condition of these stimulus-selective neurons. To evaluate the drug effects on the population of PFC cells, we derived and compared the area under the ROC curve (AUROC) as a measure of neuronal selectivity. Here, the AUROC is a nonparametric measure of the discriminability of two distributions of firing rates recorded in the stimulus present and stimulus absent conditions, respectively. Values of 0.5 indicate no separation, and values of 1 signal perfect discriminability. Figure 4 depicts example cells showing improved stimulus selectivity after blocking NMDA (MK condition; Fig. 4a,b) and GABA(A) receptors (Bic condition; Fig. $4 c, d)$. In the former case, the AUROC value increases from 0.87 in control condition to 0.97 in MK condition; and in the latter case, the AUROC value increases from 0.61 in control condition to 0.77 in Bic condition. Comparing AUROC values during drug and control conditions for the population of stimulus-selective neurons yielded significantly higher AUROC values in both drug conditions. Mean AUROC values increased from 0.71 (SE 0.01) in control to 0.74 (SE 0.01) with administration of $\mathrm{MK}\left(t_{(71)}=-2.20, p=0.031\right.$, paired $t$ test $)$, and from 0.68 (SE 0.01) in control to 0.74 (SE 0.01) with administration of Bic $\left(t_{(82)}=-4.19, p<0.001\right.$, paired $t$ test $)$. The blockade of both NMDA and GABA(A) receptors thus improved working memory selectivity to stimulus condition (Fig. 5). The distribution of 
analysis window durations during which neurons exhibited stimulus selectivity is depicted in Figure 6. For both MK (Fig. 6a) and Bic (Fig. 6b), neurons show a mixture of brief and sustained selectivity.

As every recording session started with a control block, we wanted to ensure that drug effects were not based on duration of recording time. Therefore, we compared AUROC values of the first and second control block separately for both drug conditions. AUROC values were comparable for neurons recorded in MK condition $\left(t_{(10)}=0.47, p=0.647\right.$, paired $t$ test $)$ as well as for neurons recorded in Bic condition $\left(t_{(20)}=-0.72, p=0.478\right.$, paired $t$ test).

To elucidate mechanisms that lead to enhanced selectivity, we further investigated drug effects on firing rates in the preferred and nonpreferred stimulus condition separately. For stimulusselective neurons modulated with $\mathrm{MK}$, firing rates in control trials were by definition higher for the preferred stimulus condition $(5.92 \pm 0.79)$ than for the nonpreferred condition $(2.88 \pm 0.43)$ (Fig. 7). MK did not change absolute firing rates of these neurons in either the preferred stimulus condition $\left(6.3912 \pm 0.77, t_{(71)}=\right.$ $-0.60, p=0.552$, paired $t$ test; Fig. $7 a)$ or in the nonpreferred stimulus condition $\left(2.82 \pm 0.44, t_{(71)}=0.02, p=0.981\right.$, paired $t$ test; Fig. $7 b$ ). Firing rate differences between drug and control trials for preferred $(0.47 \pm 0.85)$ and nonpreferred $(-0.06 \pm$ $0.47)$ stimulus conditions were comparable $\left(t_{(71)}=0.97, p=\right.$ 0.334 , paired $t$ test; Fig. $7 c$ ). The absence of MK effects at the population level is also depicted in Figure 8 showing the normalized spike density histograms of the population of stimulusselective neurons treated with MK. An analysis of the Fano factor, a measure of firing rate dispersion and variability, revealed no significant difference between control and MK trials for either the preferred $\left(t_{(71)}=0.287, p=0.775\right.$, paired $t$ test $)$ or the nonpreferred stimulus condition $\left(t_{(68)}=1.44, p=0.156\right.$, paired $t$ test $)$. Together, these results suggest that the improved selectivity with MK arises from a combination of nonsignificant changes in the activity of selective neurons.

Next, we analyzed how Bic achieved the improved selectivity reported above. Again, by definition, mean firing rates in control trials were higher for the preferred stimulus condition (5.67 \pm $0.94)$ than for the nonpreferred condition $(2.81 \pm 0.59)$ for stimulus-selective neurons modulated with Bic (Fig. 9). Bic did not increase absolute firing rates in either the preferred stimulus condition $\left(t_{(82)}=-1.61, p=0.111\right.$, paired $t$ test; Fig. $\left.9 a\right)$ or in the nonpreferred condition $\left(t_{(82)}=-1.27, p=0.207\right.$, paired $t$ test; Fig. 9b). A comparison of firing rate differences between control and drug trials for preferred and nonpreferred stimulus condition revealed that Bic tended to increase firing rates stronger for the preferred stimulus condition $(2.47 \pm 1.54)$ than for the nonpreferred condition $(1.24 \pm 0.97)\left(t_{(82)}=1.79, p=0.077\right.$, paired $t$ test; Fig. $9 c)$. Figure 10 depicts normalized spike density histograms of the population of stimulus-selective neurons treated with Bic that illustrate these effects across time. The Fano factors for Bic were comparable between control and drug trials, both for the preferred condition $\left(t_{(82)}=0.10, p=0.920\right.$, paired $t$ test $)$ and the nonpreferred condition $\left(t_{(79)}=0.61, p=0.547\right.$, paired $t$ test $)$.

\section{Individual neurons increased selectivity in response to both NMDA and GABA(A) receptor blockade}

To find out how individual neurons were affected by blockage of NMDA and GABA(A) receptors, we further analyzed the subset of 53 stimulus-selective neurons in sequential MK and Bic conditions. Figure $11 a-c$ depicts an example cell showing enhanced delay selectivity with administration of either drug. MK in this

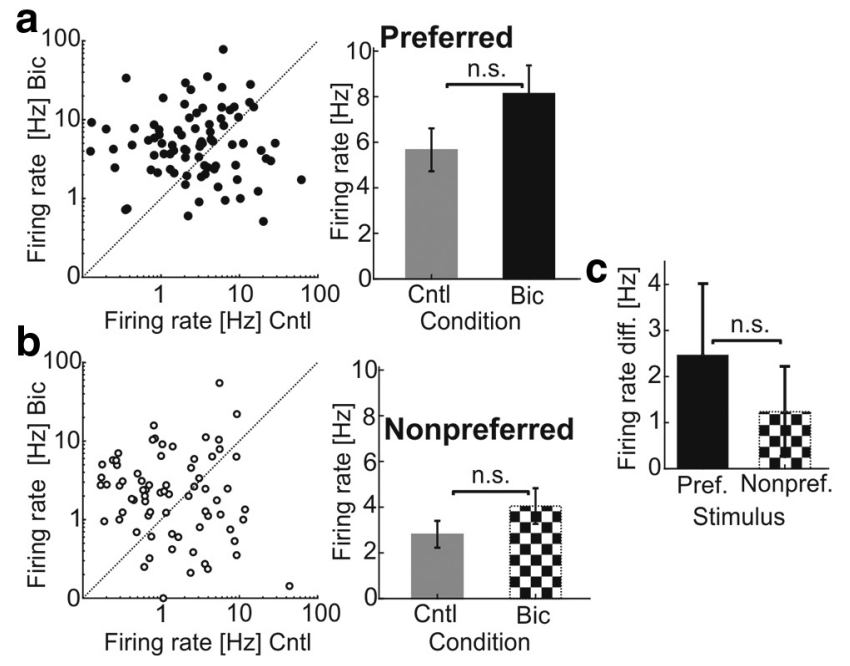

Figure 9. Bic effects on firing rate for preferred and nonpreferred stimulus condition. $\boldsymbol{a}, \boldsymbol{b}$ Firing rates for control and Bic condition separated for preferred $(\boldsymbol{a})$ and nonpreferred $(\boldsymbol{b})$ stimulus condition. Left, Firing rates for each neuron. Right, Mean firing rates for the respective population. $c$, Differences in mean firing rate between Bic and control condition for the preferred and nonpreferred stimulus condition. Error bars indicate SEs. n.s., not significant.

case increases selectivity by diminishing the firing rate stronger for the stimulus absent condition than for the stimulus present condition.

A population analysis applying again an ROC analysis of selectivity to stimulus condition during the respective analysis windows in the delay revealed that 33 of these neurons enhanced their selectivity with administration of MK, 38 did so by administration of Bic, and 28 neurons enhanced selectivity with either drug compared with control. Other possible combinations were infrequent, with 10 neurons decreasing selectivity with MK but increasing it with Bic, 5 neurons increasing selectivity with MK but decreasing it with Bic, and 10 neurons decreasing selectivity with either drug (Fig. 11d). Statistical testing revealed that the proportion of cells that improved their selectivity with both drugs $(52.83 \%)$ was within the range expected by the proportion of cells that improved their selectivity with one of the drugs (44.64\%) ( $p=0.143$, one-sided binomial test). Overall, $85 \%$ (28 of 33) of the neurons that improved their selectivity with application of MK also improved their selectivity when Bic was applied. Likewise, $74 \%$ ( 28 of 38 ) of those neurons that improved their selectivity with application of Bic also improved their selectivity when MK was applied.

Effects were comparable for the subset of BS and NS. Ten of the 53 neurons could not be classified as neither NS nor BS, 26 were BS, and 17 were NS. Of the 26 BS, 17 improved their selectivity with administration of MK, 19 did so with administration of Bic, and 13 with either drug. The proportion of BS that improved selectivity with both drugs (50.00\%) was within the range expected by the proportion of cells that improved their selectivity with one of the drugs $(47.78 \%)$ ( $p=0.489$, one-sided binomial test). Of the $17 \mathrm{NS}, 11$ improved their selectivity with administration of MK, 13 did so with administration of Bic, and 9 with either drug. Again, the proportion of NS that improved selectivity with both drugs $(52.94 \%)$ was within the range expected by the proportion of cells that improved their selectivity with one of the drugs $(49.48 \%)(p=0.483$, one-sided binomial test $)$.

\section{Discussion}

In rhesus monkeys performing a delayed decision task requiring working memory, we probed the effects of the excitatory gluta- 
matergic and inhibitory GABAergic transmitter systems on single neurons by combining single-cell recordings and simultaneous micro-iontophoretic drug applications. As major results, we found that both the blockade of excitatory glutamatergic synapses with NMDA-receptor antagonists $\mathrm{MK}$, as well as the inactivation of inhibitory synapses by GABA(A)receptor antagonist Bic increased prefrontal neurons' stimulus selectivity during the delay period, albeit only subtle for NMDA receptors. These results contrast previous findings in behaving monkeys (Rao et al., 2000; Wang et al., 2013). Moreover, we probed individual neurons not only with one of the receptor antagonists, but with both. We found that most neurons (both putative pyramidal cells as well as inhibitory interneurons) that improved their signal-to-noise ratio with one of the drugs also increased their selectivity with the other.

Performance changes by the drugs were only seen in 1 of the 2 tested monkeys; administration of either drug improved performance of Monkey Q, but not Monkey Z. Additionally, we observed that both monkeys significantly aborted more trials in drug conditions. Overall, these behavioral findings were rather unexpected, given that the effect of micro-iontophoresis is very focal (Herz et al., 1969). We thus assume that the drugs affected small clusters or micro-networks of neurons, rather than individual cells alone.

\section{Blocking NMDA receptors increases spontaneous responses and improves stimulus selectivity}

We show a significant, albeit mild reduction of spontaneous firing rate after administration of glutamatergic NMDA-receptor antagonist MK in awake behaving monkeys. Only previous in vitro studies have shown a decrease in spontaneous firing rate after administration of MK (Huettner and Bean, 1988; Rotaru et al., 2011) or studies in anesthetized rats after previous administration of NMDA (Zhang et al., 1992; Wang et al. 2013) found that iontophoretic administration of MK in awake behaving monkeys produced no significant decrease in spontaneous firing rate, albeit a marked reduction of firing rates in all task epochs for delay selective neurons. The discrepancy of our findings to Wang et al. (2013) might be explained because MK only acts on open channels, and its effectiveness thus depends on the presence of glutamate (Huettner and Bean, 1988).

Whether MK has a net excitatory or inhibitory effect on clusters of neurons should depend on the ratio of recruited pyramidal cells and interneurons and may possibly be a function of drug dosage. Indeed, high dosages of MK caused excitatory effects in rodents, whereas low dosages did not (Jackson et al., 2004). Such excitatory effects of MK are suspected to be mediated by an inhibition of glutamatergic input in inhibitory interneurons, which would lead to net disinhibition of local circuits.

An important finding of the current study is that blockade of NMDA receptors by MK improved neuronal selectivity during working memory phases. In agreement with our finding, Jackson et al. (2004) found improved working memory performance at low doses of MK in the PFC of rats. In the same study, performance was, however, impaired at high doses. Wang et al. (2013) found impaired performance as well as reduced task related firing of delay cells of monkeys in an oculomotor delayed

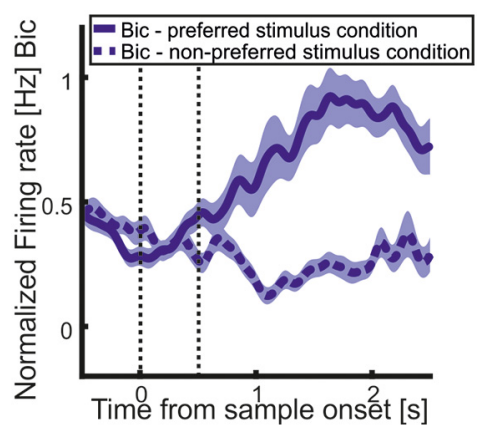
ent doses of the NMDA antagonist ketamine. In the ODR task, monkeys have to make a saccade to a remembered spatial location after a memory delay period. In this ODR task, iontophoretic application of MK mimicked the neuronal effects of systemic ketamine administration by reducing the activity of delay cells, however more so for the preferred direction. This effect caused an impairment of stimulus coding during the ODR task, as opposed to an enhancement of stimulus selectivity as observed in the current study.

The disparate findings on working memory activity in ODR tasks versus feature-based delayed response tasks may relate to anatomically distinct PFC neurons that have been described for spatial and feature-based working memory (Wilson et al., 1993), even though many single neurons represent both spatial and visual information (Rao et al., 1997). An alternative explanation is that the delay activity in the ODR task might reflect mainly motor preparation signals or allocation of spatial attention rather than maintenance of signals in working memory (Lebedev et al., 2004; Takeda and Funahashi, 2004; Markowitz et al., 2015). This is because the monkeys know from the onset of the sample location where they have to make a saccade in the subsequent test phase. In our delayed decision task, however, the monkeys lacked information during the delay period that would have allowed them to prepare an action. Thus, the ODR task might entail specific circuits engaged in preparatory motor signaling, which could be differentially modulated by glutamatergic and GABAergic receptors. Such disparate pharmacological findings on working memory activity in ODR tasks versus feature-based delayed response tasks have also been reported for the dopaminergic modulatory system (Ott and Nieder, 2017, 2019; Vijayraghavan et al., 2016; Stalter et al., 2020).

The precise mechanisms behind the increase in stimulus selectivity remains elusive. In our study, MK effects for preferred and nonpreferred stimuli were comparable, suggesting that NMDA receptors are not only active for preferred stimulus, but at least partially also for the nonpreferred stimulus. We believe that it was an interplay between modest (but in themselves nonsignificant) changes in firing rates and firing variability that added up to significant changes in selectivity as detected by the ROC analysis.

\section{Blocking GABA(A) receptors increases neuronal selectivity preferentially by disinhibiting preferred stimuli}

Iontophoretic administration of $\mathrm{GABA}(\mathrm{A})$ - antagonist Bic generally increased the spontaneous firing rates of pyramidal cells and 


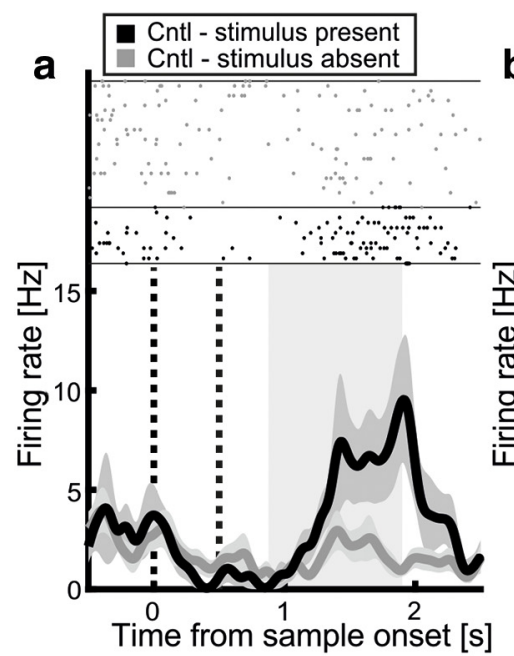

d

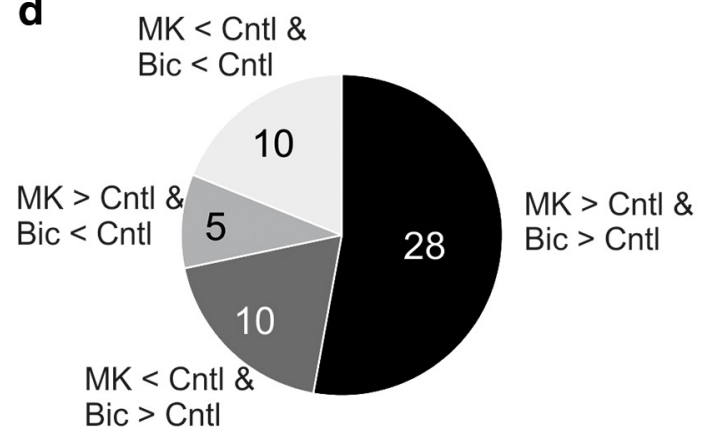

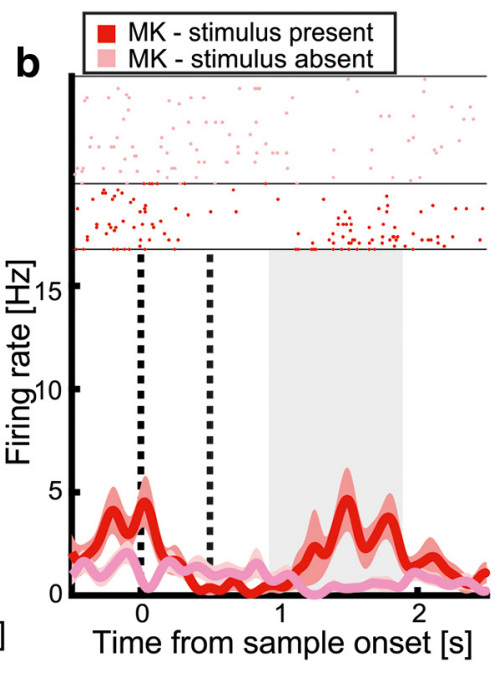

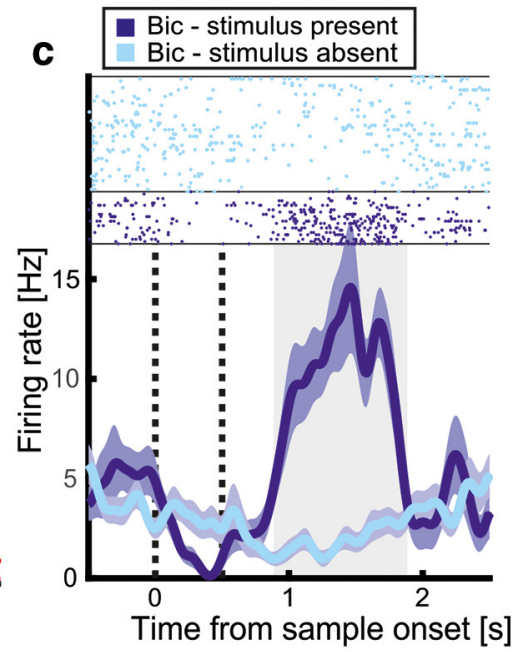

Figure 11. Stimulus-selective neurons modulated by Bic and MK. $\boldsymbol{a}-\boldsymbol{c}$, Raster plots and spike density histograms representing the activity of a broad spiking cell preferring stimulus-present trials from Monkey Q in control ( $\boldsymbol{a}$ ), Bic (b), and MK (c) conditions. Dashed vertical lines indicate beginning of stimulus presentation and delay period. Shaded backgrounds represent the analysis window. Dark colors represent stimulus-present trials. Light colors represent stimulus-absent trials. Shaded areas around the spike density histograms represent respective SEs. $\boldsymbol{d}$, Amount of neurons that increased/decreased their selectivity with drug administration. $<$, $>$, show whether the AUROC value, signifying discriminability between preferred and nonpreferred stimulus condition, was higher in the respective drug or control condition.

interneurons in lateral PFC. Bic had a stronger effect on pyramidal cells than on interneurons. Both findings are in line with similar previous studies (e.g., Rao et al., 2000).

As a major finding of the current study, blockade of GABA(A) receptors by $\mathrm{Bic}$ also improved neuronal selectivity during working memory periods. The supportive effect of blocking GABA receptors on memory is in partial agreement with behavioral studies in rodents that found improved memory retention and consolidation after administration of GABA antagonists (Luft et al., 2004; Kim et al., 2012). The blockade of GABA(A) receptors by $\mathrm{Bic}$ tended to increase the firing rate stronger for the preferred than the nonpreferred stimulus condition and thereby improved selectivity. This suggests that GABA downregulates memory processing by diminishing coding of preferred stimuli.

Contrary to our findings, Rao et al. (2000) found that iontophoretic administration of Bic in PFC of macaque monkeys performing an ODR task cancels spatial tuning of pyramidal cells and interneurons, especially in the delay phase. In their study, Bic impaired tuning by disinhibition of the nonpreferred direction as well as directions neighboring the preferred one, but also created tuning in a subset of previously untuned cells.

As discussed previously, the disparate findings in the study by Rao et al. (2000) compared with our study may be based on differences in spatial versus feature-based task protocols, as well as different types of cognitive signals (premotor vs pure working memory aspects) activated during the delay periods. In addition, GABA most likely affects working memory in a dose-dependent manner, and possibly follows an inverted- $U$ response curve. This idea is supported by the findings that too much as well as too little GABA impaired optimal behavioral performance (Pezze et al., 2014; Bast et al., 2017; Ferguson and Gao, 2018).

\section{NMDA and GABA(A) effects are mediated by the same neurons}

In previous iontophoretic studies with monkeys, only one pharmacological substance was explored per neuron. We were therefore interested to find out if and how individual neurons would react to application of both NMDA and GABA antagonists. We found that most neurons that improved their signal-to-noise ratio with one of the drugs also increased their selectivity with the other. This was true for both putative pyramidal cells and inhibitory interneurons. This leads to the conclusion that both major classes of cortical neurons contained glutamatergic NMDA and GABA receptors. The observed selectivity-promoting effects of $\mathrm{MK}$ and Bic were hence also visible at the single-cell level, so that more than half of our recorded neurons increase their selectivity with either drug.

The observed drug effects were comparable for the subset of BS and NS neurons (i.e., putative pyramidal cells and inhibitory interneurons). This is to be expected when the input of a recorded neuron is affected. However, the net output effect of both cell types on the postsynaptic neurons is expected to be in the opposite direction. Since iontophoresis does not only affect one particular neuron at the recording site but also nearby neurons as 
parts of cortical microcircuits, the observed effects may not necessarily be due to direct effects on the respective receptors of the recorded neurons but could also stem from secondary effects mediated via microcircuits. This could explain some of the variance found at the single-neuron level and emphasizes the importance of larger neuron population analyses.

Differentiating NMDA and GABA effects hence gets more complicated as glutamatergic transmission is controlled by GABA receptors; and vice versa, GABAergic transmission is controlled by NMDA receptors (Del Arco and Mora, 2002; Higley, 2014; Farahmandfar et al., 2017). Because of this delicate interplay of excitation and inhibition in cortical networks, neuropsychiatric conditions, such as schizophrenia, have also been associated with impairments in the NMDA system as well as the GABA system (Benes, 1995; Gonzalez-Burgos and Lewis, 2012; Datta and Arnsten, 2018). This suggests that a better understanding of these transmitter systems, also with respect to potential psychiatric therapies, requires an investigation of glutamatergic and GABAergic effects back-to-back.

\section{References}

Arnsten AF, Wang MJ, Paspalas CD (2012) Neuromodulation of thought: flexibilities and vulnerabilities in prefrontal cortical network synapses. Neuron 76:223-239.

Auger ML, Floresco SB (2014) Prefrontal cortical GABA modulation of spatial reference and working memory. Int. J Neuropsychopharmacol 18: pyu013.

Auger ML, Floresco SB (2017) Prefrontal cortical GABAergic and NMDA glutamatergic regulation of delayed responding. Neuropharmacology 113:10-20.

Bast T, Pezze M, McGarrity S (2017) Cognitive deficits caused by prefrontal cortical and hippocampal neural disinhibition. Br J Pharmacol 174:3211-3225.

Benes FM (1995) Altered glutamatergic and GABAergic mechanisms in the cingulate cortex of the schizophrenic brain. Arch Gen Psychiatry 52: 1015-1024.

Blum RA (1952) Effect of subtotal lesions of frontal granular cortex on delayed reaction in monkeys. Arch Neurol Psychiatry 67:375-386.

Chrobak JJ, Hinman JR, Sabolek HR (2008) Revealing past memories: proactive interference and ketamine-induced memory deficits. J Neurosci 28:4512-4520.

Cole BJ, Klewer M, Jones GH, Stephens DN (1993) Contrasting effects of the competitive NMDA antagonist CPP and the non-competitive NMDA antagonist MK 801 on performance of an operant delayed matching to position task in rats. Psychopharmacology (Berl) 111:465-471.

Compte A, Brunel N, Goldman-Rakic PS, Wang XJ (2000) Synaptic mechanisms and network dynamics underlying spatial working memory in a cortical network model. Cereb Cortex 10:910-923.

Dash PK, Moore AN, Kobori N, Runyan JD (2007) Molecular activity underlying working memory. Learn Mem 14:554-563.

Datta D, Arnsten AF (2018) Unique molecular regulation of higher-order prefrontal cortical circuits: insights into the neurobiology of schizophrenia. ACS Chem Neurosci 9:2127-2145.

Del Arco A, Mora F (2002) NMDA and AMPA/kainate glutamatergic agonists increase the extracellular concentrations of GABA in the prefrontal cortex of the freely moving rat: modulation by endogenous dopamine. Brain Res Bull 57:623-630.

Diester I, Nieder A (2008) Complementary contributions of prefrontal neuron classes in abstract numerical categorization. J Neurosci 28:77377747.

Eiselt AK, Nieder A (2013) Representation of abstract quantitative rules applied to spatial and numerical magnitudes in primate prefrontal cortex. J Neurosci 33:7526-7534.

Fano U (1947) Ionization Yield of Radiations. II. The Fluctuations of the Number of Ions. Phys Rev 72:26-29.

Farahmandfar M, Akbarabadi A, Bakhtazad A, Zarrindast MR (2017) Recovery from ketamine-induced amnesia by blockade of GABA-A receptor in the medial prefrontal cortex of mice. Neuroscience 344:48-55.

Ferguson BR, Gao WJ (2018) PV interneurons: critical regulators of E/I balance for prefrontal cortex-dependent behavior and psychiatric disorders. Front Neural Circuits 12:37.

Funahashi S, Bruce CJ, Goldman-Rakic PS (1989) Mnemonic coding of visual space in the monkey's dorsolateral prefrontal cortex. J Neurophysiol 61:331-349.

Fuster JM (2015) The prefrontal cortex, Ed 5. London: Academic.

Goldman-Rakic PS (1995) Cellular basis of working memory. Neuron 14:477-485.

Goldman-Rakic PS (1996) Regional and cellular fractionation of working memory. Proc Natl Acad Sci U S A 93:13473-13480.

Goldman-Rakic PS (1999) The "psychic" neuron of the cerebral cortex. Ann N Y Acad Sci 868:13-26.

Gonzalez-Burgos G, Lewis DA (2012) NMDA receptor hypofunction, parvalbumin positive neurons, and cortical gamma oscillations in schizophrenia. Schizophr Bull 38:950-957.

Green DM, Swets JA (1966) Signal detection theory and psychophysics. New York: Wiley.

Herz A, Zieglgänsberger W, Färber G (1969) Microelectrophoretic studies concerning the spread of glutamic acid and GABA in brain tissue. Exp Brain Res 9:221-235.

Higley MJ (2014) Localized GABAergic inhibition of dendritic $\mathrm{Ca}^{2+}$ signalling. Nat Rev Neurosci 15:567-572.

Homayoun H, Moghaddam B (2007) NMDA receptor hypofunction produces opposite effects on prefrontal cortex interneurons and pyramidal neurons. J Neurosci 27:11496-11500.

Huettner JE, Bean BP (1988) Block of N-methyl-D-aspartate-activated current by the anticonvulsant MK801: selective binding to open channels. Proc Natl Acad Sci U S A 85:1307-1311.

Jackson ME, Homayoun H, Moghaddam B (2004) NMDA receptor hypofunction produces concomitant firing rate potentiation and burst activity reduction in the prefrontal cortex. Proc Natl Acad Sci U S A 101:8467-8472.

Jacob SN, Ott T, Nieder A (2013) Dopamine regulates two classes of primate prefrontal neurons that represent sensory signals. J Neurosci 33:13724-13734

Kim DH, Kim JM, Park SJ, Cai M, Liu X, Lee S, Shin CY, Ryu JH (2012) GABA(A) receptor blockade enhances memory consolidation by increasing hippocampal BDNF levels. Neuropsychopharmacology 37:422-433.

Lebedev MA, Messinger A, Kralik JD, Wise SP (2004) Representation of attended versus remembered locations in prefrontal cortex. PLoS Biol 2:e365.

Lorrain DS, Baccei CS, Bristow LJ, Anderson JJ, Varney MA (2003) Effects of ketamine and N-methyl-D-aspartate on glutamate and dopamine release in the rat prefrontal cortex: modulation by a group II selective metabotropic glutamate receptor agonist LY379268. Neuroscience 117: 697-706.

Luft T, Pereira GS, Cammarota M, Izquierdo I (2004) Different time course for the memory facilitating effect of bicuculline in hippocampus, entorhinal cortex, and posterior parietal cortex of rats. Neurobiol Learn Mem 82:52-56.

Markowitz DA, Curtis CE, Pesaran B (2015) Multiple component networks support working memory in prefrontal cortex. Proc Natl Acad Sci U S A 112:11084-11089.

Merten K, Nieder A (2012) Active encoding of decisions about stimulus absence in primate prefrontal cortex neurons. Proc Natl Acad Sci U S A 109:6289-6294.

Miller EK, Nieder A, Freedman DJ, Wallis JD (2003) Neural correlates of categories and concepts. Curr Opin Neurobiol 13:198-203.

Mishkin M (1957) Effects of small frontal lesions on delayed alternation in monkeys. J Neurophysiol 20:615-622.

Ott T, Nieder A (2017) Dopamine D2 receptors enhance population dynamics in primate prefrontal working memory circuits. Cereb Cortex 27:4423-4435.

Ott T, Nieder A (2019) Dopamine and cognitive control in prefrontal cortex. Trends Cogn Sci 23:213-234.

Ott T, Jacob SN, Nieder A (2014) Dopamine receptors differentially enhance rule coding in primate prefrontal cortex neurons. Neuron 84:13171328.

Pezze M, McGarrity S, Mason R, Fone KC, Bast T (2014) Too little and too much: hypoactivation and disinhibition of medial prefrontal cortex cause attentional deficits. J Neurosci 34:7931-7946. 
Rao SC, Rainer G, Miller EK (1997) Integration of what and where in the primate prefrontal cortex. Science 276:821-824.

Rao SG, Williams GV, Goldman-Rakic PS (2000) Destruction and creation of spatial tuning by disinhibition: GABAA blockade of prefrontal cortical neurons engaged by working memory. J Neurosci 20:485-494.

Rotaru DC, Yoshino H, Lewis DA, Ermentrout GB, Gonzalez-Burgos G (2011) Glutamate receptor subtypes mediating synaptic activation of prefrontal cortex neurons: relevance for schizophrenia. J Neurosci 31:142-156.

Sawaguchi T, Matsumura M, Kubota K (1989) Delayed response deficits produced by local injection of bicuculline into the dorsolateral prefrontal cortex in Japanese macaque monkeys. Exp Brain Res 75:457-469.

Skoblenick K, Everling S (2012) NMDA antagonist ketamine reduces task selectivity in macaque dorsolateral prefrontal neurons and impairs performance of randomly interleaved prosaccades and antisaccades. J Neurosci 32:12018-12027.

Smith JW, Gastambide F, Gilmour G, Dix S, Foss J, Lloyd K, Malik N, Tricklebank M (2011) A comparison of the effects of ketamine and phencyclidine with other antagonists of the NMDA receptor in rodent assays of attention and working memory. Psychopharmacology (Berl) 217:255-269.

Stalter M, Westendorff S, Nieder A (2020) Dopamine gates visual signals in monkey prefrontal cortex neurons. Cell Rep 30:164-172.e4.

Suzuki Y, Jodo E, Takeuchi S, Niwa S, Kayama Y (2002) Acute administration of phencyclidine induces tonic activation of medial prefrontal cortex neurons in freely moving rats. Neuroscience 114:769-779.

Takeda K, Funahashi S (2004) Population vector analysis of primate prefrontal activity during spatial working memory. Cereb Cortex 14:1328-1339.

Thiele A, Delicato LS, Roberts MJ, Gieselmann MA (2006) A novel electrode-pipette design for simultaneous recording of extracellular spikes and iontophoretic drug application in awake behaving monkeys. J Neurosci Methods 158:207-211.

Vallentin D, Bongard S, Nieder A (2012) Numerical rule coding in the prefrontal, premotor, and posterior parietal cortices of macaques. J Neurosci 32:6621-6630.

Vijayraghavan S, Major AJ, Everling S (2016) Dopamine D1 and D2 receptors make dissociable contributions to dorsolateral prefrontal cortical regulation of rule-guided oculomotor behavior. Cell Rep 16:805-816.

Viswanathan P, Nieder A (2015) Differential impact of behavioral relevance on quantity coding in primate frontal and parietal neurons. Curr Biol 25:1259-1269.

Wang M, Arnsten AF (2015) Contribution of NMDA receptors to dorsolateral prefrontal cortical networks in primates. Neurosci Bull 31:191-197.

Wang M, Yang Y, Wang CJ, Gamo NJ, Jin LE, Mazer JA, Morrison JH, Wang XJ, Arnsten AF (2013) NMDA receptors subserve persistent neuronal firing during working memory in dorsolateral prefrontal cortex. Neuron 77:736-749.

Wang XJ (1999) Synaptic basis of cortical persistent activity: the importance of NMDA receptors to working memory. J Neurosci 19:9587-9603.

Wang XJ (2001) Synaptic reverberation underlying mnemonic persistent activity. Trends Neurosci 24:455-463.

Wang XJ (2002) Probabilistic decision making by slow reverberation in cortical circuits. Neuron 36:955-968.

Wilson FA, Scalaidhe SP, Goldman-Rakic PS (1993) Dissociation of object and spatial processing domains in primate prefrontal cortex. Science 260:1955-1958.

Yoon JH, Grandelis A, Maddock RJ (2016) Dorsolateral prefrontal cortex GABA concentration in humans predicts working memory load processing capacity. J Neurosci 36:11788-11794.

Zhang J, Chiodo LA, Freeman AS (1992) Electrophysiological effects of MK-801 on rat nigrostriatal and mesoaccumbal dopaminergic neurons. Brain Res 590:153-163. 